\title{
Advanced optical characterization of micro solid immersion lens
}

\author{
Myun-Sik Kim ${ }^{1}$, Toralf Scharf ${ }^{1}$, Mickael Brun${ }^{2}$, Segolene Olivier ${ }^{2}$, Sergio Nicoletti ${ }^{2}$, \\ and Hans Peter Herzig ${ }^{2}$ \\ ${ }^{1}$ Optics \& Photonics Technology Laboratory, Ecole Polytechnique Fédérale de Lausanne (EPFL), \\ Neuchâtel, CH-2000, Switzerland \\ ${ }^{2}$ CEA-Leti, Minatec Campus, 17 rue des Martyrs, 38054 Grenoble Cedex 9, France
}

\begin{abstract}
We report on the advanced optical characterizations of microfabricated solid immersion lenses with 2- $\mu \mathrm{m}$ diameter, operating at $\lambda=642 \mathrm{~nm}$. The main feature, the spot size reduction, has been investigated by applying a focused Gaussian beam of NA $=0.9$. Particular illuminating beams, e.g., Bessel-Gauss beams of the zeroth and the first order, a doughnutshape beam and its decompositions, i.e. two-half-lobes beams, have also been used to influence the shape of the immersed focal spot. Detailed optical characterizations have been conducted by measuring the amplitude and phase distributions with a high-resolution interference microscope (HRIM) in volume around the focal spot. The immersion effect of the $\mathrm{SiO}_{2}$ solid immersion lens leads to a spot-size reduction of approximately 1.5 which agrees well with theory. Particularly shaped incident beams exhibit a comparable size reduction of the immersed spots. Such structured focal spots are of significant interest in optical trapping, lithography, and optical data storage systems.
\end{abstract}

Keywords: Solid immersion lens (SIL), illumination engineering, beam shaping, azimuthal polarization, Bessel-Gauss beam, Laguerre-Gaussian beam, doughnut beam, high-resolution interference microscope (HRIM).

\section{INTRODUCTION}

For the ideal lens, a focus can be defined by marginal rays with a half angle $\theta$ as shown in Fig. 1(a). The mechanism of focusing by lenses is the refraction of light. The measure of performance of a lens is the numerical aperture ${ }^{1}$ (NA) and is defined as

$$
N A=n \cdot \sin \theta,
$$

where $\theta$ is the angle the ray makes with the optical axis (half the value of the angle of the focusing cone), and $n$ is the refractive index of the medium through which the rays pass. If we insert a high refractive index medium with a planar surface in order to increase the NA by factor of $n$ as illustrated in Fig. 1(b), a refraction effect reduces the focusing angle $\theta$ by the law of refraction (Snell's Law) ${ }^{2}$

$$
\frac{\sin \theta_{1}}{\sin \theta_{2}}=\frac{n_{2}}{n_{1}}
$$

where $\theta_{1}$ and $\theta_{2}$ are incident and refraction angles and $n_{1}$ and $n_{2}$ are refractive indices of incident and refraction media, respectively. The NA is therefore sustained as before. However, aberrations due to the form of the lens degrade the quality of the focus. It was R. Hooke who first discussed the immersion technique to overcome such effects and to improve the imaging performance in microscopy in $1678^{3}$. Although the concept of homogeneous immersion had preceded Abbe's pioneering works, he constructed the first oil-immersion lens when setting up the imaging theory of microscopy ${ }^{4}$. In a homogeneous immersion concept, oil serves as a perfect index matching medium for the cover glass, so that complete homogeneity is obtained as shown in Fig. 1(c). Finally, the numerical aperture is increased by a factor equal to the refractive index $n$ and calculated by Eq. (1).

*myunsik.kim@epfl.ch; phone 41-32-718-3279; fax 41-32-718-3201; http://opt.epfl.ch/

Optical Micro- and Nanometrology IV, edited by Christophe Gorecki, Anand K. Asundi, Wolfgang Osten, Proc. of SPIE Vol. 8430, 84300E - (C) 2012 SPIE · CCC code: 0277-786X/12/\$18 - doi: 10.1117/12.921871 


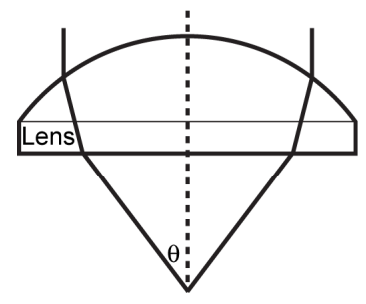

(a)

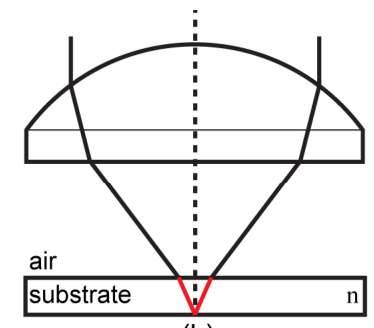

(b)

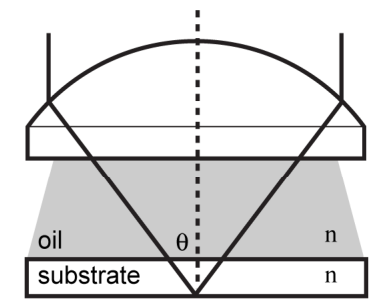

(c)

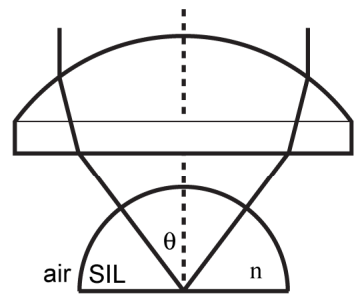

(d)

Figure 1. Working principal of the immersion schemes: (a) focusing in air, (b) focusing through a substrate with a planar interface, (c) liquid immersion by oil, and (d) solid immersion by a hemispherical solid medium. The numerical aperture (NA) is a product of the refractive index $(n)$ and the sine of the half-focusing angle $(\sin \theta)$ [see Eq. (a)].

In 1990, Mansfield et al. suggested a new immersion concept illustrated in Fig. 1(d), which he named solid immersion lens $(\mathrm{SIL})^{5}$. Figure 1(d) shows that focusing on the center of the hemispherical solid leads to normal incidence with respect to the spherical interface. Therefore, there is no additional refraction at the interface between air and a solid medium. The immersion leads to an increase of the NA by a factor of $n$ (the refractive index of the solid medium). For a long time the fabrication of SILs was limited to macro-size, i.e. millimeter range dimensions, due to the lack of advanced fabrication technologies. The advance of micro- and nano-technology boosts the developments of different types of solid immersion lenses, for instance, diffractive SILs ${ }^{6}$, micro-size SILs ${ }^{7,8}$, nano-size SIL-like elements ${ }^{9,10}$, and subwavelengthsize SILs ${ }^{11}$. The reported micro-size SILs in Refs. 7 and 8 are still relatively large $(>\sim 10 \mu \mathrm{m})$. When the SILs are large, the fabrication and alignment errors produce substantial aberrations because the amount of aberrations is proportional to the size of the optical elements. Therefore, when the characteristic size of the SIL becomes smaller and falls within the wavelength size, aberrations, especially the spherical aberration, become negligible ${ }^{11}$. In the visible, the size of a few micrometers is a good target value to avoid aberration effects and at the same time to facilitate the fabrication processes. By using conventional micromachining processes, hemispherical $\mu$-SILs of $2-\mu \mathrm{m}$ diameter and filled with $\mathrm{SiO}_{2}$ have been fabricated ${ }^{12}$. The optical characterizations of such small objects are not trivial. Furthermore, the verification of the immersion effect requires super-resolution methods like scanning near-field optical microscopy (SNOM) or an equivalent immersion technique in the observation system, for example, using another SIL facing to the target SIL (specimen). In either case, the alignment of the focused beam on the micro-size SIL and the observation system is a very difficult task and 3D optical field measurements as well as investigations of the phase are not straightforward.

The goal of this paper is to report on advanced optical characterizations of such small $\mu$-SILs by using a high-resolution interference microscope. The HRIM facilitates such complex alignments by the in situ monitoring of the illumination beam. The liquid immersion technique, which employs an oil $\left(n_{\text {oil }}=n_{S I L}\right)$ for $\mathrm{SiO}_{2} \mu$-SILs, allows the 3D field measurements of the immersed spots through the $\mu$-SIL. Thanks to the interferometric function of the HRIM, we can investigate not only the amplitude and but also the phase distributions. The transverse phase distribution is utilized to verify the size of the Airy disc rather than the full-width at half maximum (FWHM) spot size. In addition, we report on the feasibility of particular beam shaping of the immersed focal spot by applying Bessel-Gauss beams of the $0^{\text {th }}$ and the $1^{\text {st }}$ orders, a doughnut-shape beam and its decompositions as an illumination beam ${ }^{13}$. Strong confinement of such structured focal spots is of significant interest in several applications, for example, optical trapping of relatively small particles, direct laser write (DLW) lithography, and optical data storage systems.

\section{EXPERIMENTAL CONDITIONS}

\subsection{Experimental setup}

The high-resolution interference microscope has already been proven as a powerful tool for the 3D characterizations of macro-, micro-, and nano-optical elements ${ }^{13-18}$ and to measure light field features with nanometric precision ${ }^{19}$. The HRIM is a kind of holographic microscopy in a sense of recording interferograms and retrieving information of light fields of interest, especially amplitude and phase distributions. As it relies on interferometry, such as a Mach-Zehnder interferometer and phase shifting interferometry (PSI), we have named our experimental system an interference microscope. In general, interferometric testing systems have been developed for two-dimensional (2D) measurements, for example, surface profiling and wavefront measurements of light fields in a plane of interest ${ }^{20}$. The major difference 
from other types of interference microscopes is the capability to record real 3D amplitude and phase fields by scanning the sample together with illumination elements along the optical axis (the $z$-axis in Fig. 2).

The HRIM operates in transmission with in-line geometry by employing a Mach-Zehnder interferometer as shown in Fig. 2. A single mode polarized laser diode (CrystaLaser, $642 \mathrm{~nm}$ : DL640-050-3) is employed as light source, which is expanded and collimated by a spatial filtering technique. This illumination plane wave, which is normally incident upon the illumination element, in our case, the $\mathrm{NA}=0.9$ focusing objective, propagates along the positive $z$-axis and is polarized in the $x$-direction. In the classical interferometric arrangement a polarizing beam splitter (PBS) divides intensities in a reference and an object arm with adjustable energy ratio. Half wave plates (HWP) and Glan-Taylor (G-T) polarizers are used to adjust the intensities and to optimize the contrast of the interference fringes. In the reference arm, a piezo-electrically driven mirror is mounted to change the optical path length. The phase distribution in a single plane of the wave field is obtained by measuring the interference fringes at different mirror positions and employing the wellknown 5-frame algorithm, which is called Schwider-Hariharan method ${ }^{21,22}$. Five frames of the intensity pattern, from which the 2D phase information can be directly retrieved, are recorded with each frame being shifted by adding an optical path of $\lambda / 4$ or $0.5 \pi$. The sample and the illumination element are mounted on a precision piezo stage with a $z$-scan range of $500 \mu \mathrm{m}$ and a nominal accuracy of $1 \mathrm{~nm}$ (MAD LAB CITY, NANO Z500). This $z$-axis piezo stage is used to precisely define the plane of interest at the highest resolution and measure 3D light fields through the $\mu$-SIL by scanning the SIL and the focusing lens along the axial direction, as shown in Fig. 2.

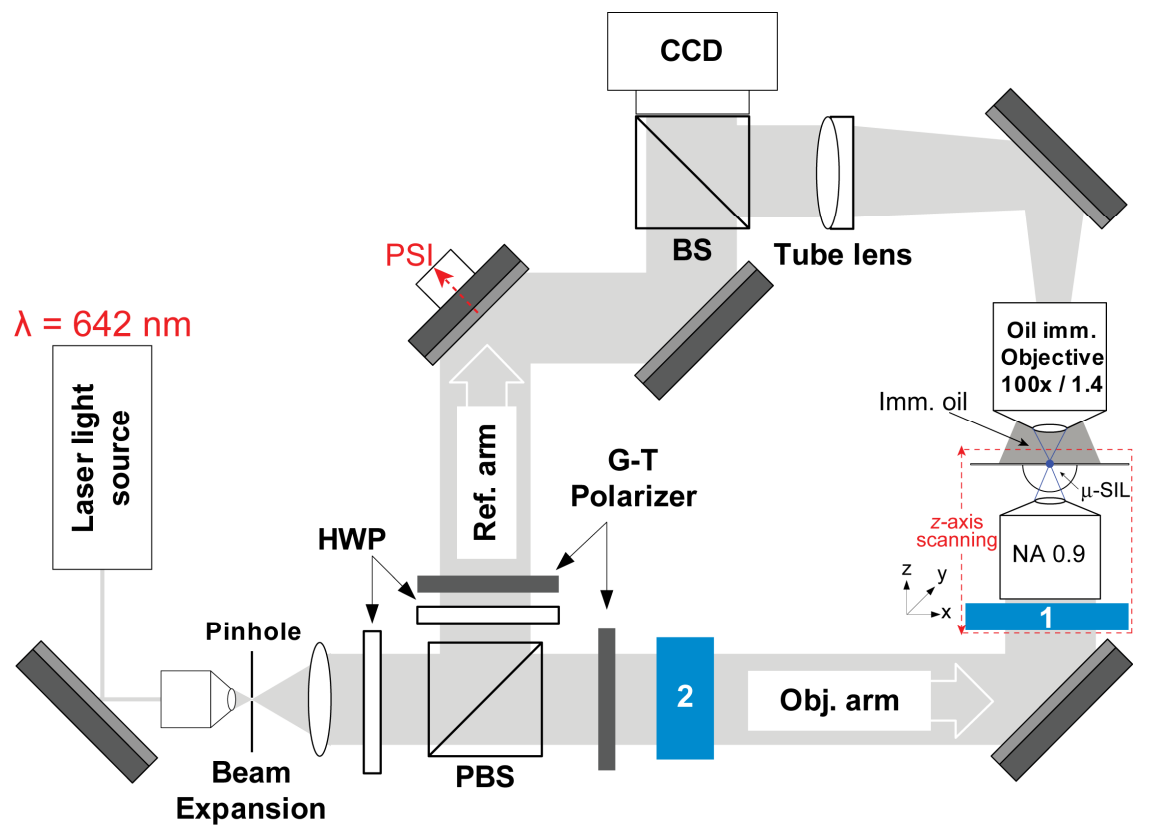

Figure 2. Schematic of the experimental setup of the high-resolution interference microscope, when the $\mu$-SIL and an NA0.9 focusing lens are inserted on the sample stage ( $z$-axis piezo actuator). In the reference arm the piezo driven mirror modulates the optical path with 5 steps of $\lambda / 4$ which leads to the 5 -step phase shifting interferometry (PSI). In the object arm, a plane wave emerges to the focusing lens at normal incidence. Scanning the $\mu$-SIL together with the focusing lens allows the 3D amplitude and phase measurements. In the object arm, additional elements to manage the wavefront and polarization can be used to manipulate the incident illumination. They are placed either in to the position of Squares 1 or 2. The details of such illumination elements will be discussed in Section 2.2.

\subsection{Illumination engineering}

Under standard measurement conditions, an expanded collimated beam which resembles a plane wave with linear polarization parallel to the $x$-axis illuminates the focusing lens as depicted in Fig. 2. By introducing additional illumination components, we generate particular illumination beams, such as Bessel Gauss beams, a doughnut-shape beam and two-half-lobes beams. For example, when the central part of a plane-wave illumination is blocked by an annular aperture, which is inserted in square 1 of Fig. 2, the amplitude distribution in the focal plane resembles a zerothorder Bessel function of the first kind in radial direction within finite extensions ${ }^{23,24}$. The focusing causes a Gaussian apodization in the amplitude distribution that can be represented as a product of a Bessel function and a Gaussian profile. 
This type of experimental Bessel beams are known as Bessel-Gauss beams ${ }^{25}$ or a focused Bessel beams. Unlike a diffraction-free beam ${ }^{26,27}$, such beam carries a finite power and transverse and longitudinal extensions due to the fast decrease of the Gaussian profile. Figure 3(a) shows the generated $0^{\text {th }}$ order Bessel-Gauss beam by an NA=0.9 focusing lens with an annular illumination ${ }^{13}$. A radial/azimuthal polarization converter, which is placed in Square 2 of Fig. 2, shifts the incident linear polarization to the radial or azimuthal polarization ${ }^{28}$. Such cylindrically symmetric polarization incidences lead to the doughnut-mode beams, i.e. Laguerre-Gaussian (LG) mode beams ${ }^{29}$, when they are focused by a lens. Focusing LG beams by a lens with an annular slit ${ }^{30}$ or by an axicon lens ${ }^{31}$ are well-known methods to produce higher order Bessel beams. In this work, we focus the annular beam, which leads to the Bessel-Gauss beam, of the azimuthal polarization in order to generate the $1^{\text {st }}$ order Bessel-Gauss beam, in other words, azimuthal Bessel-Gauss beam ${ }^{32}$. The generated $1^{\text {st }}$ order Bessel-Gauss beam is shown in Fig. 3(b). When the azimuthal polarization incidence is focused by an NA=0.9 objective lens, the doughnut-shape beam is formed [see Fig. 3(c)]. Such a doughnut beam can be decomposed by a linear polarizer, and the results are shown in Figs. 3(d) and 3(e) as the two-half-lobes spot. The extinction axis in the transverse plane is parallel to the principal axis of the polarizer. These five particular beams will be applied to the beam shaping of the immersed foal spots in Section 4.

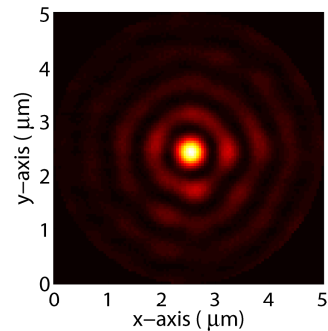

(a)

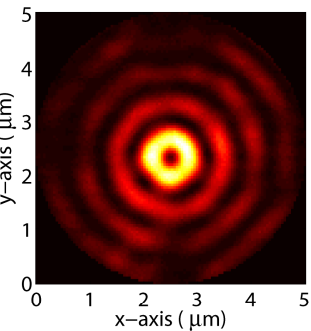

(b)

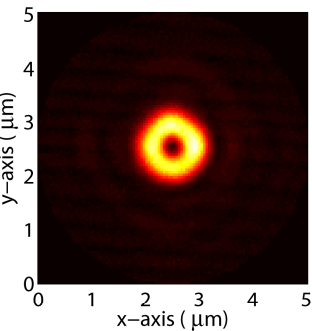

(c)

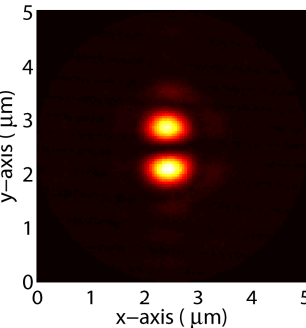

(d)

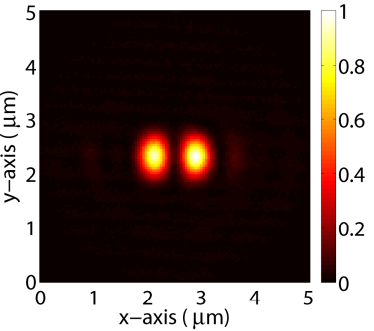

(e)

Figure 3. Measured transverse intensity distributions of the particularly shaped beams in the focal plane of a NA=0.9 objective. (a) The $0^{\text {th }}$ order Bessel-Gauss beam by focusing the annular illumination of the linear polarization and (b) the $1^{\text {st }}$ order Bessel-Gauss beam by focusing the annular illumination of the azimuthal polarization. Focusing an azimuthally polarized beam with and without an additional polarizer leads to vortex beams. (c) A typical doughnut-shape beam without a polarizer. A polarizer parallel to (d) the $x$ - and (e) $y$-axes decomposes such a doughnut beam to the two-half-lobes spots with the extinction axis parallel to the direction of the polarizer. Image size is $5 \times 5 \mathrm{~m}^{2}$. Intensities are all normalized.

\subsection{Geometry of $\mu$-SIL}

The conventional micromachining processes, such as, etching and deposition, are applied to fabricate the $\mu$-SILs. The details of the fabrication processes are reported elsewhere ${ }^{12}$. Figure 4 shows the schematic geometry of the fabricated $\mu$ SIL. The hemispherical SIL body $\left(\mathrm{SiO}_{2}\right)$ of $2-\mu \mathrm{m}$ diameter is hold by the $200 \mathrm{~nm}$-thick $\mathrm{Si}_{3} \mathrm{~N}_{4}$ membrane. The main SIL chip is formed in a 550- $\mu \mathrm{m}$ thick 100 silicon ( $\mathrm{Si}$ ) substrate, which is back-side etched to release the spherical surface to air. The $\mu$-SILs are designed in array with various numbers, e.g., $3 \times 3,2 \times 2$, and $1 \times 6$, and a single SIL as well. Microscopic photographs of the $\mu$-SILs arranged in $2 \times 2$ array are shown in Fig 5. Since the thin $\mathrm{Si}_{3} \mathrm{~N}_{4} \mathrm{membrane}$ is transparent for the visible spectrum, we can still see and localize the $\mu$-SILs through the membrane as shown in Fig. 5(b). In this way, optical characterizations are carried out through this membrane in the HRIM, as illustrated in Fig. 2.

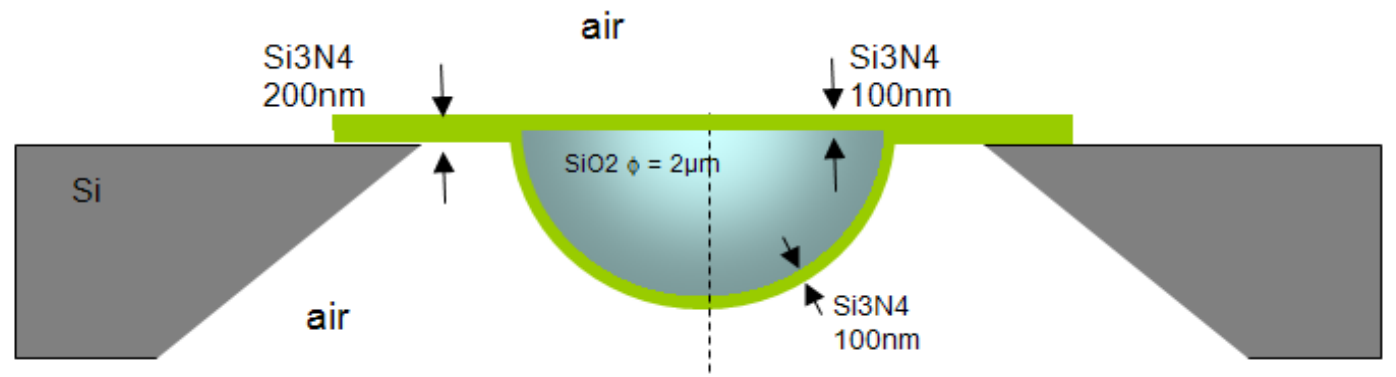

Figure 4. Schematic geometry of the $\mu$-SIL structure: the hemispherical SIL body $\left(\mathrm{SiO}_{2}\right)$ of $2-\mu \mathrm{m}$ diameter is hold by the 200 -nm thick $\mathrm{Si}_{3} \mathrm{~N}_{4}$ membrane. 


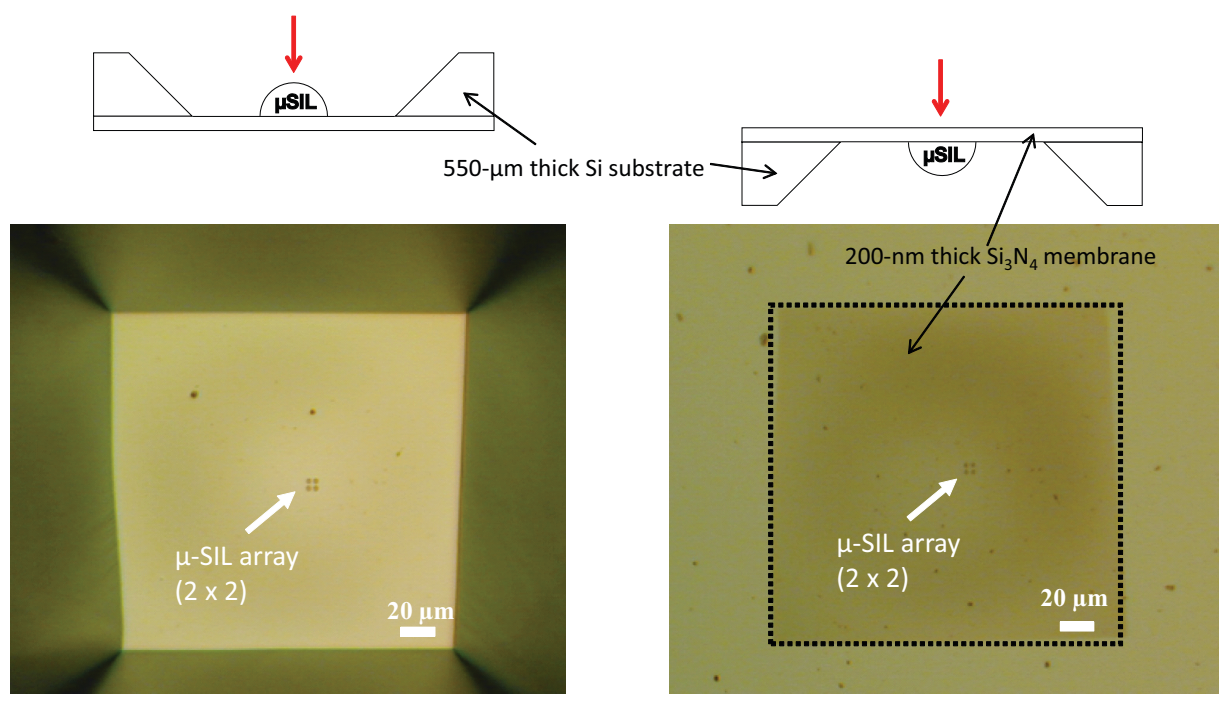

Figure 5. Microscopic photographs of the $2 \times 2 \mu$-SIL array: (left) the view from the SIL side and (right) the view through the $\mathrm{Si}_{3} \mathrm{~N}_{4}$ membrane. Four circular features are the $\mu$-SILs of $2-\mu \mathrm{m}$ diameter. The red arrows indicate the direction of the observation.

\section{FUNDAMENTAL OPTICAL CHARACTERIZATIONS}

Fundamental function of the SILs is to reduce the focal spot due the immersion effect, which automatically increases the resolution in the microscopy and lithography applications. In this section we verify the spot-size reduction by applying a highly focused linearly polarized beam on the SIL. Here, we do not use any additional illumination elements like a polarization converter and an annular aperture. The incident plane wave is focused by an NA=0.9 objective lens. Figure 6 shows the focal spots on the bottom of the SIL chip, where the nitride membrane holds the $\mu$-SILs. The $3 \times 3$ circular features represent the bottom view of the $2-\mu \mathrm{m}$ SILs. When we focus the light on the $\mathrm{Si}_{3} \mathrm{~N}_{4}$ membrane, as shown in Fig. 6(a) [out of the SIL], the size of the focal spot is set to the reference that represents the non-immersed spot. The $3 \times 3$ array of the SILs facilitates the statistical study of uniformity and homogeneity of the SIL characteristics without moving the chip. Now, we move this reference focal spot on each SILs at each time, as shown in Figs. 6(b) - 6(h) [on the SIL]. They demonstrate the spot-size reduction and uniformity of the optical response of the SILs.

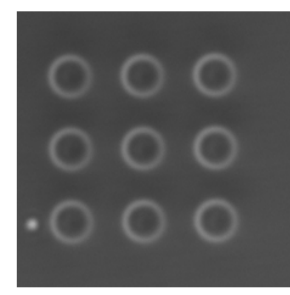

(a)

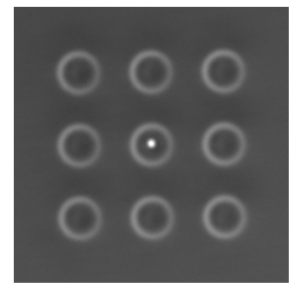

(e)

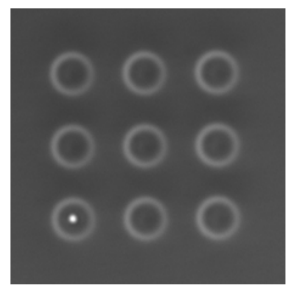

(b)

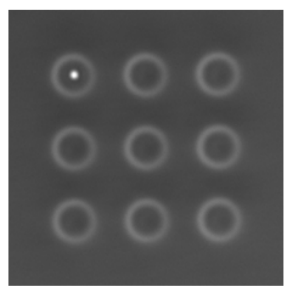

(f)

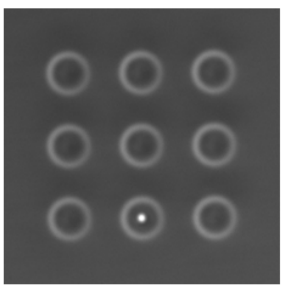

(c)

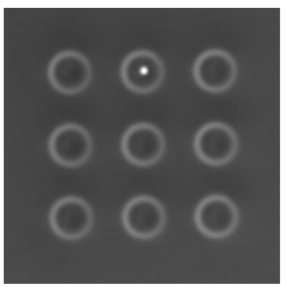

(g)

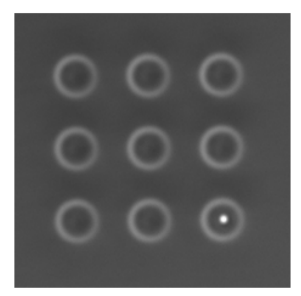

(d)

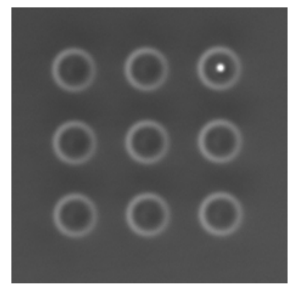

(h)

Figure 6. Focal spots on the bottom of the SIL chip, where the $3 \times 3$ circular features represent the array of the SILs. (a) The focal spot out of the SIL [reference: non-immersed spot], which is seen in the lower left corner of the image, and (b) - (h) the focal spots on the individual SILs [immersed spots]. 
Further quantitative analysis has been carried out by using measured 3D data as shown in Fig. 7, which contain quantitative information of the spot size and the peak intensity of each spot. Here, we exemplarily present data for the non-immersed spot of Fig. 6(a) and the immersed spot of Fig. 6(b). While the amplitude data show the FWHM spot sizes and the peak intensity, the phase data reveal the Airy disc size, which is isolated by the circular phase singularity line at the focus. The phase measurement demonstrates the reduction of the full spot size, i.e. the Airy disc size.
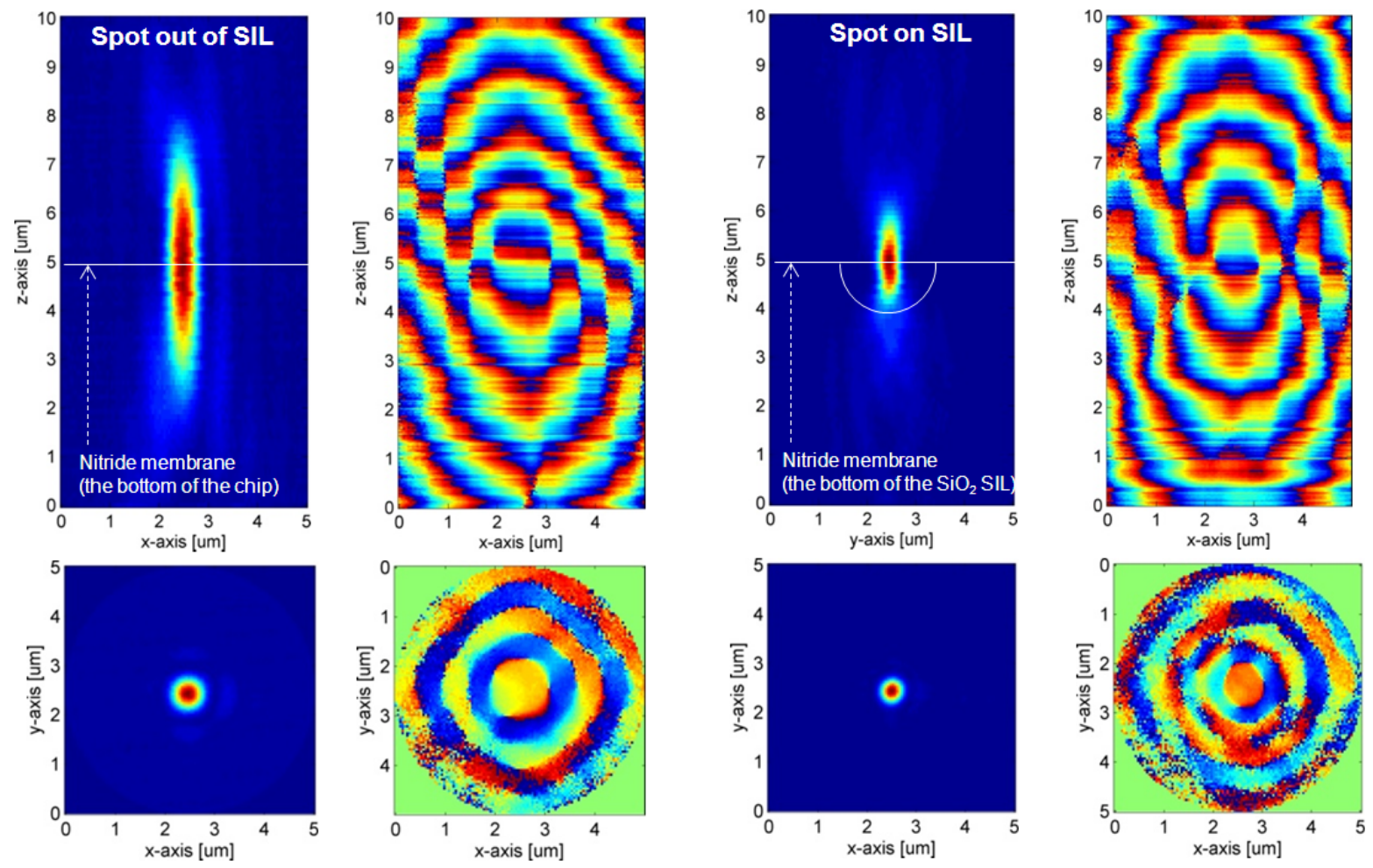

(a)

(b)

Figure 7. The measured 3D amplitude and phase distributions of (a) the non-immersed spot [Fig. 6(a)] and (b) the immersed spots [Fig. 6(b)]. The upper row shows the $x-z$ distributions and the lower row shows the $x-y$ distributions. While the amplitude data show the FWHM spot sizes and the peak intensity, the phase data reveal the Airy disc size, which is isolated by the circular phase singularity line at the focus.

For statistical analysis, the reference spot [see Fig. 6(a)] has been measured five times at the same position. The transverse spot sizes are measured to be $502 \pm 10 \mathrm{~nm}$ along the $x$-axis and $504 \pm 9 \mathrm{~nm}$ along the $y$-axis. The longitudinal spot size and the peak intensity are found to be $3805 \pm 39 \mathrm{~nm}$ and $115 \pm 4$ [arb. unit], respectively. The small standard deviation values confirm the repeatability and reliability of the measurement. For the immersed spots, the standard deviation from five measurements exhibits analogous results to that of the non-immersed spot. We highlight the in situ comparison of one single measurement of each SIL from the $3 \times 3$ array [see Figs. 6(b) - 6(h)]. Table 1 shows the spot sizes in the transverse and longitudinal directions and the peak intensity of the immersed spots by each SIL. In this case, the small standard deviation of each value confirms the uniformity of the performance of each SIL in the same chip. When comparing the average transverse size of the non-immersed and the immersed spots, we find the ratio of the transverse spot-size reduction is approximately 1.5 [x-axis: 1.47 and $y$-axis: 1.4$]$. This agrees with the refractive index of the $\mathrm{SiO}_{2}$ SIL, which is 1.5 at $642 \mathrm{~nm}$ wavelength. Moreover, the reduction of the Airy disc size is also found to be approximately 1.5 , as seen in the transverse phase distributions in Fig. 7. The peak intensity is enhanced approximately $50 \%$ by the immersion effect, which is due to stronger spatial confinement of light. We analyze the result by using the power of the Gaussian beam, which can be expressed as

$$
P=\frac{1}{2} \pi w^{2} I,
$$


where $I$ is the peak intensity and $w$ is the beam waist ${ }^{33}$. For the case of no absorption and loss $[P=$ constant $]$, the peak intensity value is proportional to the square of the spot size. The spot size reduction of 1.5 theoretically leads to the peak intensity enhancement of $2.25\left(=1.5^{2}\right)$. In reality, the peak intensity is enhanced by a factor of 1.87 . This indicates there is a transmission loss of $17 \%$, which shows a good agreement with the measured transmission loss of $14 \%$.

Table 1. Spot size and peak intensity of the immersed spots shown in Figs. 6(b) - 6(h).

\begin{tabular}{|c|c|c|c|c|}
\hline SIL & $x$-axis [nm] & $y$-axis [nm] & $z$-axis [nm] & $\begin{array}{c}\text { Peak intensity } \\
{[\text { arb. unit] }}\end{array}$ \\
\hline (b) & 351 & 372 & 1435 & 217 \\
\hline (c) & 337 & 361 & 1408 & 209 \\
\hline (d) & 343 & 350 & 1392 & 204 \\
\hline (e) & 347 & 357 & 1452 & 225 \\
\hline (f) & 336 & 353 & 1291 & 225 \\
\hline (g) & 335 & 356 & 1495 & 213 \\
\hline (h) & 338 & 356 & 1406 & 212 \\
\hline Average & $\mathbf{3 4 1}$ & $\mathbf{3 5 8}$ & $\mathbf{1 4 1 1}$ & $\mathbf{2 1 5}$ \\
\hline $\begin{array}{l}\text { Standard } \\
\text { deviation }\end{array}$ & 6.1 & 7.1 & 63.4 & 7.9 \\
\hline
\end{tabular}

\section{BEAM SHAPING OF THE IMMERSED SPOT}

Apart from the focused linearly polarized beam, we apply particularly shaped beams in order to demonstrate beam shaping of an immersed spot. As we have shown in Section 2.2, we can generate the Bessel-Gauss beam of the $0^{\text {th }}$ order and the $1^{\text {st }}$ order and the doughnut-shape beam and its decompositions. For reference we use the non-immersed spot and focus the engineered illumination beam on the nitride membrane. Figure 8 shows the results of the Bessel-Gauss beams of the 0th and 1st orders. The upper row shows the CCD images, where we can see the bottom of the $\mu$-SIL and the engineered illumination spot. The non-immersed spots in Figs. 8(a) and 8(c) are larger than the immersed spots in Figs. 8(b) and 8(d). Figure 9 shows the results of the illuminations of the doughnut beam and its decompositions. The case of the 0th order Bessel-Gauss beam exhibits the spot size reduction of approximately 1.43, which agrees well with the result of Section 3. The qualitative comparisons in Figs. 8 and 9 demonstrate that the immersed spots are smaller than the nonimmersed spots for all the cases. However, the cases of the 1st order Bessel-Gauss beam, the doughnut beam and the two-half-lobes beams show a spot size reduction ratio smaller than that of the refractive index of the SIL. We guess that the relatively large transverse size of such beams (approximately $1.5 \mu \mathrm{m}$ ) prevents the $2-\mu \mathrm{m}$ hemispherical SIL body properly accommodating such a large spot.
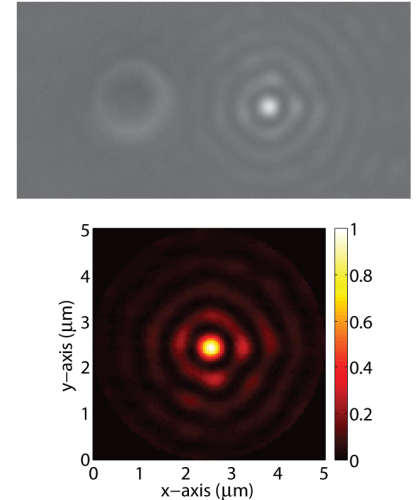

(a)
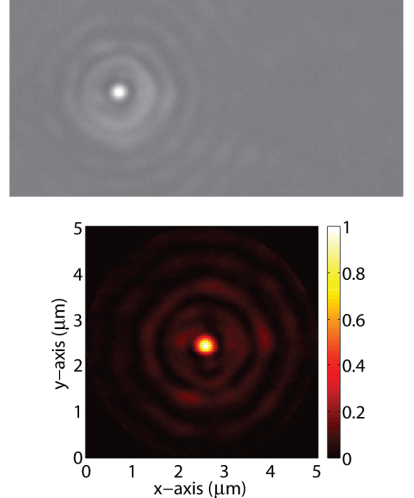

(b)
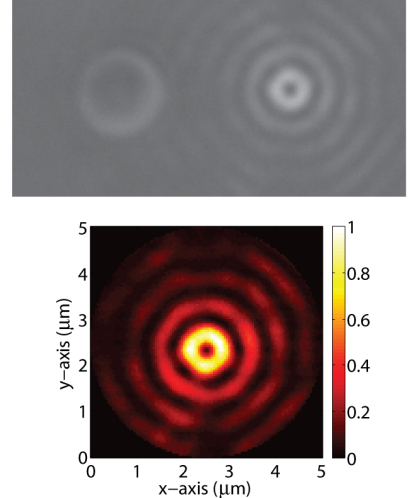

(c)
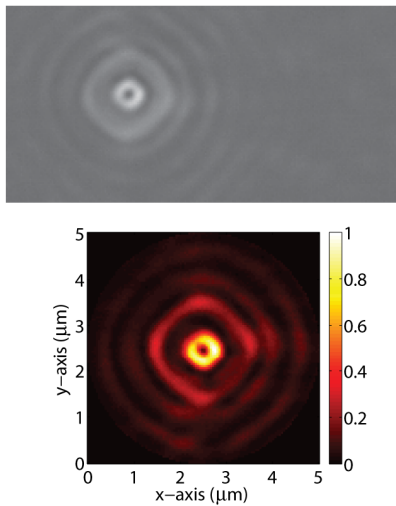

(d)

Figure 8. Results of the Bessel-Gauss beam illuminations. The upper row shows the CCD images, where the circular feature in the left-hand side represents the bottom of the $\mu$-SIL and the engineered focal spot is captured together with the SIL. The lower row shows the measured transverse intensity distributions of the non-immersed and immersed spots, where intensities are all normalized. (a) The non-immersed and (b) the immersed spots of the Bessel-Gauss beam of the $0^{\text {th }}$ order. (c) The non-immersed and (d) the immersed spots of the Bessel-Gauss beam of the $1^{\text {st }}$ order. 

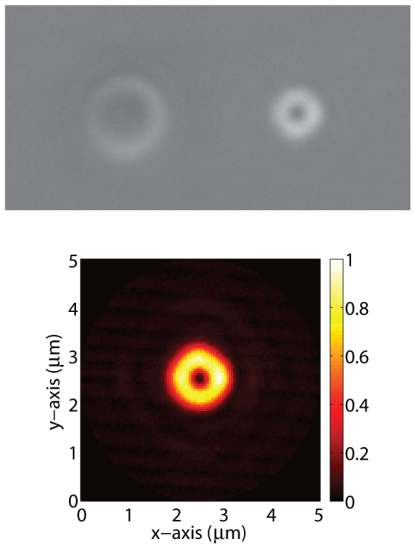

(a)
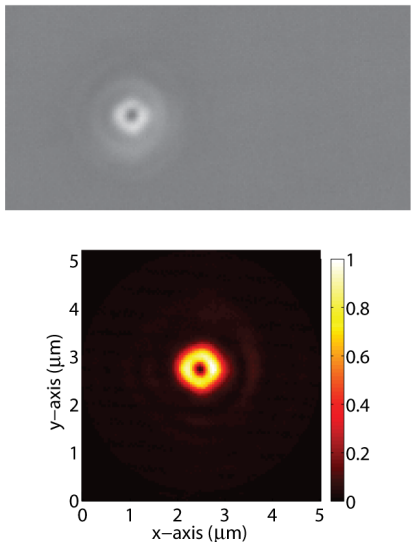

(b)
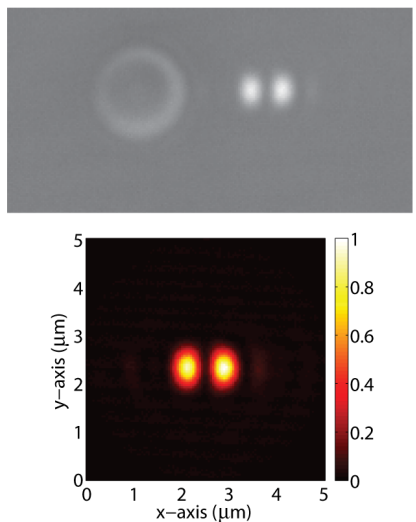

(e)
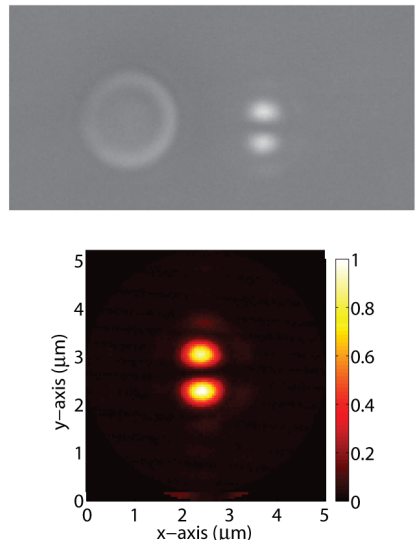

(c)

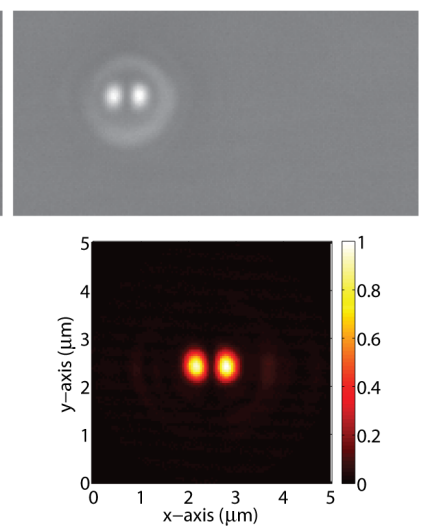

(f)
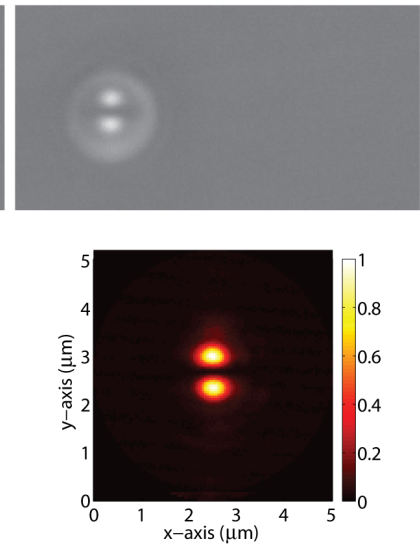

(d)

Figure 9. Results of the illuminations of the doughnut beam and its decompositions. The upper row shows the CCD images, where the circular feature in the left-hand side represents the bottom of the $\mu$-SIL and the engineered focal spot is captured together with the SIL. The lower row shows the measured transverse intensity distributions of the non-immersed and immersed spots, where intensities are all normalized. (a) The non-immersed and (b) the immersed spots of the doughnut beam. (c) The non-immersed and (d) the immersed spots of the decomposed doughnut beam, by a polarizer parallel to the $x$-axis. (d) and (e) show the results of the decomposed beam by a polarizer parallel to the $y$-axis.

\section{CONCLUSIONS}

We report on optical characterizations of microfabricated solid immersion lenses ( $\mu$-SILs) with 2- $\mu$ m diameter, at visible wavelength $(\lambda=642 \mathrm{~nm})$. Since aberrations are not limiting the performance of such small SILs, they are basically more robust to the fabrication and alignment errors. The fundamental characteristic, which is the spot size reduction, has been investigated by applying a focused Gaussian beam of NA $=0.9$. For the optical characterizations, the HRIM, which incorporates a 100X/NA1.4 oil immersion objective and a Mach-Zehnder interferometer, is employed to monitor illuminating beams and measure the output spots emerging from the SILs. The 3D amplitude and phase distributions of the focal spots with and without the $\mu$-SILs reveal the information of the FWHM and the full spot (Airy disc) sizes. Analysis of the 3D intensity data provides not only the transverse spot size but also the longitudinal spot size, and moreover the peak intensity enhancement that is caused by a tighter focus. Both the transverse FWHM and full spot sizes are reduced by a factor of 1.5 , which agrees well with the refractive index of the $\mathrm{SiO} 2$. The enhancement of the peak intensity is theoretically proportional to the square of the spot size reduction ratio when there are no loss and absorption. The peak intensity is $50 \%$ increased, which is 1.87 times larger than that of the non-immersed spot. Moreover, we found there is approximately $17 \%$ transmission loss through the $\mu$-SIL, which is small enough to be negligible in the applications.

Particular illuminating beams have also been applied for the beam-shaping of the immersed spots. First, the BesselGauss beams of the zeroth order and the first order are generated by focusing the annular illumination beams of a linear 
polarization and an azimuthal polarization. Second, a focused beam with an azimuthal polarization leads to a doughnutshape hollow focus. By decomposing such a doughnut beam with a linear polarizer, two-half-lobes beams are produced. The immersion effect of the $\mu$-SIL reduces such beams of particular shapes. The shape of the spot is maintained but the size is reduced due to the corresponding immersion effect. Such particularly structured beams are of significant interest for various applications. For example, the hollow beams are useful for the optical trapping of specific specimens that might be repelled and pushed away from the regions of maximum intensity ${ }^{34}$. Low refractive-index particles, such as air bubbles in liquid, and metallic particles at certain frequencies are typically repelled by the bright spot ${ }^{35,36}$. Absorbing (i.e. non-transparent) particles in liquids and gaseous medium are also repelled and pushed away from intensity maxima ${ }^{37-39}$. In addition, a dark trap is more beneficial for some applications than a bright trap for a simple reason: the light may not interact with the trapped object. This is of great advantage while trapping photosensitive materials, such as biological cells, and for trapping neutral atoms ${ }^{40-42}$. Thanks to the long focal depth, the Bessel beam plays an important role in the bright trap scheme as well ${ }^{43}$. Tighter confinement of the structured beams can be applied to increase the resolution of lithography and optical data storage systems.

\section{ACKNOWLEDGMENT}

The research leading to these results has received funding from the European Community's Seventh Framework Programme FP7-ICT-2007-2 under grant agreement No. 224226.

\section{REFERENCES}

[1] Abbe, E., "On the Estimation of Aperture in the Microscope," J. of the Royal Microscopical Society 2 1, 388-423 (1881).

[2] Born, M. and Wolf, E., [Principles of Optics $7^{\text {th }}$ Ed.], Cambridge University Press, Chap. 1 (1999).

[3] Hooke, R., [Lectures and Collections; Microscopium], Royal Society, London, 1 (1678).

[4] Abbe, E., "On New Methods for Improving Spherical Correction applied to the Construction of Wide-angled Object-glasses," J. of the Royal Microscopical Society 2, 812-824 (1879).

[5] Mansfield, S. M. and Kino, G. S., "Solid immersion microscope," Appl. Phys. Lett. 57, 2615 (1990).

[6] Brunner, R., Burkhardt, M., Pesch, A., Sandfuchs, O., Ferstl, M., Hohng, S. and White, J. O., "Diffraction-based solid immersion lens," J. Opt. Soc. Am. A 21, 1186-1191 (2004).

[7] Lang, M., Milster, T. D., Aspnes, E., Minamitani, T. and Borek, G., "Investigation of micro solid immersion lens mounting systems," Jpn. J. Appl. Phys. 46, 3737-3740 (2007).

[8] Kishia, T., Shibata, S. and Yano, T., "Fabrication of high-refractive-index glass micron-sized solid immersion lenses by a surface-tension mold technique," J. Non-Crystalline Solids 354, 558-563 (2008).

[9] Lee, J. Y., Hong, B. H., Kim, W. Y., Min, S. K., Kim, Y., Jouravlev, M. V., Bose, R., Kim, K. S., Hwang, I. C., Kaufman, L. J., Wong, C. W., Kim, P. and Kim, K. S., "Near-field focusing and magnification through selfassembled nanoscale spherical lenses," Nature 460, 498-501 (2009).

[10] Mason, D. R., Jouravlev, M .V. and Kim, K. S., "Enhanced resolution beyond the Abbe diffraction limit with wavelength-scale solid immersion lenses," Opt. Lett. 35, 2007-2009 (2010).

[11] Kim, M.-S., Scharf, T., Haq, M. T., Nakagawa, W. and Herzig, H. P., "Subwavelength-size solid immersion lens," Opt. Lett. 36, 3930-3932 (2011).

[12] Brun, M., Richard, M. and Nicoletti, S., "Integrated Micro Solid Immersion Lens for Near Field Optical Data Storage," International Symposium on optical memory (ISOM09), Mo-E-04 (2009).

[13] Kim, M.-S., Scharf, T., Mühlig, S., Rockstuhl, C. and Herzig, H. P., "Engineering photonic nanojets,” Opt. Express 19, 10206-10220 (2011).

[14] Kim, M.-S., Scharf, T. and Herzig, H. P., "Amplitude and Phase Measurements of Highly Focused Light in Optical Data Storage Systems,” Jpn. J. Appl. Phys. 49, 08KA03 (2010).

[15] Kim, M.-S., Scharf, T. and Herzig, H. P., "Small size microlenes characterization by Multiwavelength High Resolution Interference Microscopy," Opt. Express 18, 14319-14329 (2010).

[16] Kim, M.-S., Scharf, T., Mühlig, S., Rockstuhl, C. and Herzig, H. P., "Gouy Phase Anomaly in Photonic Nanojets," Appl. Phys. Lett. 98, 191114 (2011).

[17] Kim, M.-S., Scharf, T., Menzel, C., Rockstuhl, C. and Herzig, H. P., "Talbot images of wavelength-scale amplitude gratings," Opt. Express 20, 4903-4920 (2012).

[18] Kim, M.-S., Scharf, T., Etrich, C., Rockstuhl, C. and Herzig, H. P., "Longitudinal-differential interferometry: Direct imaging of axial superluminal phase propagation,” Opt. Lett. 37, 305-307 (2012). 
[19] Rockstuhl, C., Märki, I., Scharf, T., Salt, M., Herzig, H. P. and Dändliker, R., "High Resolution Interference Microscopy: A Tool for Probing Optical Waves in the Far-Field on a Nanometric Length Scale," Current Nanoscience 2, 337-350 (2006).

[20] Malacara, D., [Optical Shop Testing, 3rd ed.], WILEY, chap. 16 (2007).

[21] Schwider, J., Burow, R., Elssner, K.-E., Grzanna, J., Spolaczyk, R. and Merkel, K., "Digital wave-front measuring interferometry: some systematic error sources," Appl. Opt. 22, 3421-3432 (1983).

[22] Hariharan, P., Oreb, B. F. and Eiju, T., "Digital phase-shifting interferometry: a simple error-compensating phase calculation algorithm," Appl. Opt. 26, 2504-2506 (1987).

[23] Born, M. and Wolf, E., [Principles of Optics $7^{\text {th }}$ Ed.], Cambridge University Press, Chap. 8 (1999).

[24] Singer, W., Totzeck, M. and Gross, H., [Handbook of Optical Systems Vol. 2], Wiley,Chap. 25 (2005).

[25] Gori, F., Guattari, G. and Padovani, C., "Bessel-Gauss beams," Opt. Comm. 64, 491 (1987).

[26] Durnin, J., Miceli, J. J. and Eberly, J. H., "Diffraction-free beams," Phys. Rev. Lett. 58, 1499-1501 (1987).

[27] Indebetouw, G., "Nondiffracting optical-fields - some remarks on their analysis and synthesis," J. Opt. Soc. Am. A 6, 150-152 (1989).

[28] Stalder, M. and Schadt, M., "Linearly polarized light with axial symmetry generated by liquid-crystal polarization converters," Opt. Lett. 21, 1948 (1996).

[29] Zhan, Q., "Cylindrical vector beams: from mathematical concepts to applications," Adv. Opt. Photon. 1, 1 (2009).

[30] Vasilyeu, R., Dudley, A., Khilo, N., and Forbes, A., "Generating superpositions of higher-order Bessel beams," Opt. Express 17, 23389-23395 (2009).

[31] Arlt, J. and Dholakia, K. "Generation of high-order Bessel beams by use of an axicon," Opt. Comm. 177, 297-301 (2000).

[32] Jordan, R. H. and Hall, D. G., "Free-space azimuthal paraxial wave equation: the azimuthal Bessel-Gauss beam solution," Opt. Lett. 19, 427-429 (1994).

[33] Siegman, A. E., [Lasers], University Science books, 665 (1986).

[34] Dienerowitz, M., Mazilu, M. and Dholakia, K., "Optical manipulation of nanoparticles: a review,” J. Nanophoton. 2, 021875 (2008).

[35] Lankers, M., Popp, J., Urlaub, E., Stahl, H., Rössling, G. and Kiefer, W., "Investigations of multiple component systems by means of optical trapping and Raman spectroscopy,” J. Mol. Struct. 348, 265-268 (1995).

[36] Sasaki, K., Koshioka, M., Misawa, H., Kitamura, N. and Masuhara, H., "Optical trapping of a metal particle and a water droplet by a scanning laser beam," Appl. Phys. Lett. 60, 807-809 (1992).

[37] Davis, E. J. and Schweiger, G., [The Airborne Microparticle: Its Physics, Chemistry, Optics, and Transport Phenomena], Springer, 780-785 (2002).

[38] Shvedov, V. G., Desyatnikov, A. S., Rode, A. V., Krolikowski, W. and Kivshar, Yu. S., "Optical guiding of absorbing nanoclusters in air," Opt. Express 17, 5743-5757 (2009).

[39] Shvedov, V. G., Hnatovsky, C., Rode, A. V. and Krolikowski, W., "Robust trapping and manipulation of airborne particles with a bottle beam," Opt. Express 19, 17350-17356 (2011).

[40] Chu, S., "The manipulation of neutral particles," Rev. Mod. Phys. 70, 685-706 (1998).

[41] Isenhower, L., Williams, W., Dally, A. and Saffman, M., "Atom trapping in an interferometrically generated bottle beam trap," Opt. Lett. 34, 1159-1161 (2009).

[42] Xu, P., He, X., Wang, J. and Zhan, M., "Trapping a single atom in a blue detuned optical bottle beam trap," Opt. Lett. 35, 2164-2166 (2010).

[43] Arlt, J., Garces-Chavez, V., Sibbett, W. and Dholakia, K., "Optical micromanipulation using a Bessel light beam," Opt. Comm. 197, 239-245 (2001). 